\title{
Pons Varolii
}

National Cancer Institute

\section{Source}

National Cancer Institute. Pons Varolii. NCI Thesaurus. Code C12511.

The middle portion of the brainstem located between the midbrain and the medulla oblong ata. The fourth ventricle lies dorsal to the pons which also contains the motor trigeminal nuclei and the abducens nuclei. The cerebellum contributes a large number of afferent fibers to the pons. 